\title{
AVALIAÇÃO VOCAL DE CRIANÇAS DISFÔNICAS PRÉ E PÓS INTERVENÇÃO FONOAUDIOLÓGICA EM GRUPO: ESTUDO DE CASO
}

\section{Evaluating the dynamic vocal dysphonic children in a pre and post intervention speech therapy group: a case study}

\author{
Vanessa Veis Ribeiro (1), Ana Paula Dassie Leite (2), Bruno Leonardo Freire de Alencar (3), \\ Denise lenk Bail ${ }^{(4)}$, Maria Fernanda Bagarollo ${ }^{(5)}$
}

\begin{abstract}
RESUMO
Este estudo tem como objetivo caracterizar a dinâmica vocal de crianças disfônicas pré e pós-terapia fonoaudiológica em grupo por meio de avaliação perceptivo-auditiva e acústica da voz. Participaram seis crianças, dois meninos e quatro meninas, com idades entre sete e dez anos, com diagnóstico de disfonia funcional ou organofuncional. As crianças foram submetidas à anamnese, análise perceptivo-auditiva e acústica da voz, antes e após processo terapêutico grupal semanal, num total de doze sessões de quarenta minutos cada. Como estratégias terapêuticas foram propostas atividades de dramatizações, desenhos, brincadeiras, elaboração de painéis e realizados exercícios vocais (de forma coletiva e lúdica). Buscou-se a troca de experiências entre os membros do grupo, a construção conjunta de conhecimentos sobre a produção da voz e saúde vocal, e, a atuação direta por meio de técnicas e exercícios. Os dados foram analisados usando um nível de significância de 0,10 . Quanto aos parâmetros de rugosidade e grau global da disfonia da avaliação perceptivo-auditiva, houve diferença entre as avaliações realizadas antes e depois do processo terapêutico grupal ( $p=0,024$ e $p=0,074$, respectivamente). Em relação à análise acústica da voz pré e pós-terapia, não houve diferença para frequência fundamental e intensidade vocal média ( $p=0,288$ e $p=0,906$, respectivamente). Já para as medidas de ruído, jitter e shimmer, houve diferença entre as avaliações iniciais e finais $(p=0,079$ e $p=0,046$, respectivamente). A terapia fonoaudiológica em grupo promove modificações na dinâmica vocal de crianças disfônicas, no que se refere aos parâmetros perceptivo-auditivos e acústicos.
\end{abstract}

DESCRITORES: Disfonia; Distúrbios da Voz; Fonoterapia; Prática de Grupo; Criança

(1) Fonoaudióloga; Discente de Mestrado em Distúrbios da Comunicação Humana pela Universidade Federal de Santa Maria - UFSM, Santa Maria, RS, Brasil.

(2) Fonoaudióloga; Professora Assistente do Departamento de Fonoaudiologia da Universidade Estadual do Centro Oeste - UNICENTRO, Irati, PR, Brasil; Doutoranda em Saúde da Criança e do Adolescente pela Universidade Federal do Paraná - UFPR; Mestre em Saúde da Criança e do Adolescente pela Universidade Estadual de Campinas - UNICAMP.

(3) Otorrinolaringologista; Mestre em Cirurgia pelo Instituo de Pesquisas Médicas do Paraná - IPEM.

(4) Fonoaudióloga; Professora Colaboradora do Departamento de Fonoaudiologia da Universidade Estadual do Centro Oeste - UNICENTRO, Irati, PR, Brasil; Mestre em Distúrbios da Comunicação pela Universidade Tuiuti do Paraná - UTP;Especialização em Voz pelo CEFAC.

\section{INTRODUÇÃO}

A atuação terapêutica na Fonoaudiologia ainda é bastante voltada para o atendimento individualizado. No entanto, um novo paradigma vem trazendo transformações no campo da saúde, deslocando o eixo patologia/tratamento/controle/

(5) Fonoaudióloga; Professora Assistente do Departamento de Fonoaudiologia da Universidade Estadual do Centro Oeste - UNICENTRO, Irati, PR, Brasil; Doutora em Saúde da Criança e do Adolescente pela Universidade Estadual de Campinas - UNICAMP; Mestre em Educação pela Universidade Metodista de Piracicaba.

Conflito de interesses: inexistente 
prevenção de doenças para o eixo saúde/promoção da saúde. Nesse novo paradigma, a Promoção da Saúde se constitui no eixo norteador de toda e qualquer prática em saúde, incluindo o trabalho clínico e trazendo mudanças na sociedade a partir de (re) significações dos conceitos de saúde e doença. A saúde passa a ser vista como um processo dinâmico, uma dimensão da qualidade de vida, recurso para enfrentar e responder aos desafios da vida, e, nesse meio, a linguagem e a comunicação passam a impactar de forma intensa a saúde e, consequentemente, a vida das pessoas ${ }^{1}$.

Nesse contexto de reflexões sobre inovadoras maneiras de entender a prática clínica, surge a terapia grupal na Fonoaudiologia, que é vista como educativa e terapêutica, potencialmente eficiente pelo fato de proporcionar a construção conjunta de conhecimentos entre os sujeitos. Além disso, este tipo de prática permite trocas de experiências e anseios, que de individuais, passam a ser compartilhados por todos os integrantes do grupo, permitindo a eles a (re) significação dos seus processos patológicos, e, consequentemente, a melhora na percepção sobre sua saúde e qualidade de vida ${ }^{2}$.

$\mathrm{Na}$ área da voz, o trabalho fonoaudiológico em grupo surgiu como forma de agrupar a demanda e suprir as listas de espera. Porém, essa motivação para prática grupal está ultrapassada. Agora, o grupo passa a ser considerado um importante lugar de trocas, vivências culturais e partilha de conhecimentos, e, por isso uma forma potente de intervenção $0^{2,3}$.

Tradicionalmente, a terapia fonoaudiológica na área de voz baseava-se em oferecer modelos vocais ao paciente, buscando propiciar respostas mais próximas ao seu padrão vocal natural. Pela diferença de idade do paciente e do terapeuta, e também pela diferença de gênero, tais modelos vocais muitas vezes são inadequados ${ }^{4}$.

A perspectiva atual da terapia vocal com crianças não prioriza tal terapêutica, voltando os esforços do clínico para sensibilizar a criança em relação à sua alteração vocal e às suas expectativas, principalmente no que diz respeito às características do seu comportamento vocal ${ }^{5}$. De modo geral, a criança muitas vezes não é consciente da natureza do seu problema ou até mesmo de que sua voz esteja alterada ${ }^{4,5}$. Sendo assim, o grande desafio para o trabalho terapêutico com crianças é fazer com que conceitos complexos e abstratos sejam aprendidos de forma efetiva pelo jovem paciente, o que é difícil pelo simples fato deles serem crianças, não aceitando orientações e muitas vezes não sendo disciplinadas para realizar as atividades propostas. Todos esses fatores tornam o trabalho terapêutico vocal com crianças um grande desafio, havendo uma grande dificuldade de fazer com que elas venham a aderir ao tratamento, levando-o para a vida real e ultrapassando as barreiras da clínica ${ }^{4}$.

Em crianças, a disfonia é caracterizada pela alteração de alguns parâmetros vocais que resultam em várias modificações na qualidade do som, no timbre, no pitch ou na intensidade ${ }^{2,6}$. Sua etiologia é variada, e compreende desde afecções autolimitadas, como laringites agudas virais, até lesões incapacitantes e com risco de vida, como os tumores ${ }^{6}$. É necessário que se considerem os fatores predisponentes ou agravantes das disfonias infantis, entre os quais se podem destacar os hábitos vocais inadequados, alguns fatores biopsicossociais, a estrutura da personalidade, a inadaptação fônica e os fatores alérgicos.

Pouco se conhece sobre a produção vocal infantil, etapa em que ocorrem transformações na laringe das crianças $^{7,8}$. Por isso, torna-se instigante e desafiador analisar a voz da criança, em períodos pequenos de tempo, durante todo seu desenvolvimento ${ }^{8,9}$. Sendo assim, torna-se necessário que o profissional da área de voz avalie, diagnostique e acompanhe a evolução do seu paciente, buscando adequar suas condutas para obter melhores resultados ${ }^{7}$.

Para aprofundar os estudos da voz, o conhecimento sobre a acústica e o entendimento da fisiologia tornam-se essenciais para descrever as características de uma voz. Para isso, tem sido utilizados métodos de avaliação perceptivo-auditiva e acustica ${ }^{8-10}$. A avaliação perceptivo-auditiva é uma análise subjetiva muito utilizada, considerada padrão ouro na detecção de distúrbios vocais. A avaliação acústica refere-se a medidas objetivas, computadorizadas, que complementam a análise auditiva e fornecem parâmetros de normalidade e alteração que podem ser comparados durante todo o processo terapêutico ${ }^{7-11}$. Os parâmetros perceptivo-auditivos e acústicos auxiliam o clínico durante todo o processo terapêutico e, juntamente com os instrumentos de autoavaliação, permitem que se faça um panorama geral sobre a evolução do sujeito ao final da terapia.

Embora tenha havido um aumento das propostas de intervenções em grupo na Fonoaudiologia, os trabalhos sobre intervenções terapêuticas grupais com crianças ainda constituem um número muito pequeno quando comparados às intervenções terapêuticas vocais individuais. Tal dado indica carência e a necessidade de desenvolvimento de novos trabalhos acerca desse tema. Diante do exposto, o presente estudo teve como objetivo caracterizar a dinâmica vocal de crianças disfônicas por meio de avaliação perceptivo-auditiva e acústica da voz, pré e pós-terapia fonoaudiológica em grupo. 


\section{APRESENTAÇÃO DO CASO CLÍNICO}

Trata-se de um relato de caso clínico, referente ao processo terapêutico grupal de seis crianças disfônicas. Quanto ao gênero, participaram quatro meninas e dois meninos, com faixa etária entre sete e dez anos (média 8,33 anos), que aguardavam na lista de espera para serem atendidas em uma Clinica-Escola de Fonoaudiologia de uma cidade do interior do Estado do Paraná.

Foram incluídas crianças disfônicas, com hipótese diagnóstica fonoaudiológica de disfonia funcional ou organofuncional, que haviam passado por avaliação laringológica prévia, e possuíam diagnóstico ORL de lesão/alteração compatível com os tipos de disfonia mencionados acima. Foram adotados como critérios de exclusão: apresentar patologia de origem neurológica, auditiva, sindrômica ou qualquer outra doença que pudesse interferir no processo de fonação; ter realizado tratamento anterior para disfonia; apresentar qualidade vocal exclusivamente soprosa de grau discreto na avaliação perceptivo-auditiva inicial (tal característica é comum em crianças devido ao processo de desenvolvimento laríngeo); apresentar disfonia de causa orgânica; apresentar quaisquer outros problemas de saúde; fazer uso de medicação controlada diária. Quanto ao diagnóstico otorrinolaringológico (ORL), uma criança possuía nódulos nas pregas vocais, duas possuíam diagnóstico de fenda paralela, uma de fenda triangular médio-posterior, uma apresentou diagnóstico interrogado de ponte de mucosa, e, uma não possuía alteração laríngea.

Os dados foram coletados na Clínica-Escola de Fonoaudiologia da UNICENTRO, Irati-PR. Para analisar a efetividade do processo grupal foi realizada avaliação perceptivo-auditiva da voz e análise acústica vocal, pré e pós processo terapêutico. As avaliações foram realizadas individualmente.

Para a avaliação perceptivo-auditiva da voz, forma coletadas as amostras de emissão sustentada da vogal / $/ /$, contagem de números de 1 a 10 e as frases propostas no protocolo Consenso da Avaliação Perceptivo Auditiva da Voz (CAPE-V) elaborado pela $\mathrm{ASHA}^{12}$. O CAPE-V é um protocolo que contém escala analógico-visual, composto por uma escala analógica linear que vai de 0 a 100 $\mathrm{mm}$. Todas as emissões foram captadas em local silencioso, com um microfone unidirecional, auricular, condensador, posicionado a $45^{\circ}$ de angulação em relação à comissura labial conectado diretamente no computador e as crianças permaneceram sentadas. Todas as amostras foram gravadas em $\mathrm{CD}$, sem identificação do sujeito, em ordem aleatória, com repetição de aproximadamente 25\% (para análise da confiabilidade intra-avaliador). Posteriormente, foram encaminhadas a um fonoaudiólogo especialista em voz, com experiência em avaliação de vozes infantis. O especialista analisou as vozes de acordo com os parâmetros do CAPE-V (grau geral da disfonia, soprosidade, rugosidade, tensão, pitch e loudness). O avaliador demonstrou muito boa confiabilidade interna (cálculo do coeficiente de Kappa - MDA entre 0 e 0,5).

Para a análise acústica foi utilizado o software VOXMETRIA, da CTS Informática. Os parâmetros vocais analisados foram: frequência fundamental, jittere shimmer. A média da frequência fundamental, o jitter e o shimmer foram analisados durante a produção de emissão sustentada da vogal $/ \varepsilon /$. Já a intensidade média foi calculada durante a contagem de números de 1 a 10 . Os valores foram classificados como "normais" ou "alterados" conforme a classificação de normalidade do programa utilizado, que é de $0,6 \%$ para jitter e $6,5 \%$ para shimmer. A coleta das amostras para análise acústica e perceptivo-auditiva foi realizada nos seguintes momentos: aproximadamente uma semana antes do início do processo terapêutico e uma semana após o seu término.

O processo terapêutico foi realizado em um único grupo, e teve duração de 12 sessões, de 40 minutos cada, uma vez por semana, totalizando cerca de três meses. Durante as sessões foram propostas atividades de dramatizações, desenhos, brincadeiras, elaboração de painéis, dentre outras, buscando a troca de experiências e a construção conjunta de conhecimentos sobre a produção da voz e saúde vocal. Exercícios vocais foram realizados de forma lúdica, priorizando contextos que fossem significativos para as crianças. Os pais foram orientados a acompanhar a execução dos exercícios em casa, 3 vezes ao dia. Para controlar a realização diária dos exercícios em casa, haviam linhas na parte posterior da folha de exercícios, onde os pais e o paciente deveriam colocar a data e assinar ao lado, todas as vezes que os exercícios fossem realizados. Em todas as sessões as crianças também deveriam contar quantas vezes realizaram na semana, apontar possíveis dificuldades e/ou facilidades para a realização. Cada exercício deveria ser repetido por 15 séries. Ao final de todas as sessões os exercícios eram relembrados com as crianças, e elas eram novamente orientadas sobre a sua execução para que pudessem realizar em casa durante a semana. As técnicas realizadas ao longo do processo terapêutico foram: técnica de sons nasais, técnica de sons vibrantes, técnica de som basal, técnica de bocejo-suspiro, técnica de voz salmodiada, técnica de sons fricativos. Das 12 sessões, nove foram realizadas com as crianças e 
três com os pais (início, meio e término da terapia). As atividades realizadas em cada sessão estão dispostas na Figura 1.

Essa pesquisa foi aprovada pelo Comitê de Ética em Pesquisa da Universidade Estadual do Centro-Oeste, sob o número № 246/2010. Todos os pais ou responsáveis pelos participantes assinaram o Termo de Consentimento Livre e Esclarecido (TCLE), de acordo com a Resolução 196/96 da Comissão Nacional de Ética em Pesquisa (CONEP).

Os dados coletados foram tabulados e as variáveis foram analisadas estatisticamente por meio de testes paramétricos "t-Student Pareado" e "Igualdade de Duas Proporções", para comparação dos resultados antes e após processo terapêutico. Foi adotado nível de significância de $10 \%$, ou seja, todos os intervalos de confiança do trabalho foram construídos com $90 \%$ de confiança estatística.

\begin{tabular}{|c|c|c|}
\hline Sessões & úblico & Atividades \\
\hline 1a sessão & Pais & $\begin{array}{l}\text { Primeiro contato do grupo de pais, introdução dos conceitos de saúde } \\
\text { vocal, orientações sobre a condução do processo terapêutico e } \\
\text { esclarecimento de dúvidas }\end{array}$ \\
\hline $2^{\underline{a}}$ sessão & Crianças & $\begin{array}{l}\text { Primeiro contato do grupo, apresentação e diálogo entre os participantes, } \\
\text { orientações sobre a condução do processo terapêutico e introdução dos } \\
\text { conceitos de voz e saúde vocal }\end{array}$ \\
\hline $3^{\text {a }}$ sessão & Crianças & $\begin{array}{l}\text { Atividade de desenho e pintura, representativos da voz, com posterior } \\
\text { discussão sobre as produções de cada criança. Introdução de conceitos } \\
\text { sobre produção vocal, funções da voz, ressonância, intensidade, } \\
\text { articulação e frequência utilizando-se vídeos com personagens de } \\
\text { desenho, cantores e artistas }\end{array}$ \\
\hline $4^{a}$ sessão & Crianças & $\begin{array}{l}\text { Discussão sobre hábitos de saúde vocal e introdução dos exercícios } \\
\text { vocais: duas listas de exercícios diferentes, descritos de forma lúdica, } \\
\text { elaboradas de acordo com a patologia: fenda e nódulo* }\end{array}$ \\
\hline $5^{\mathrm{a}}$ sessão & Crianças & $\begin{array}{l}\text { Montagem de painéis com figuras: abuso vocal/cuidado com higiene } \\
\text { vocal. Posterior discussão e troca de experiências anteriores trazidas } \\
\text { pelas crianças** }\end{array}$ \\
\hline $6^{\underline{a}}$ sessão & Crianças & $\begin{array}{l}\text { Introdução dos conceitos de OFAs, CPFA e fisiologia da respiração por } \\
\text { meio de vídeos ilustrativos com posterior discussão entre as crianças e a } \\
\text { terapeuta* }^{\star \star}\end{array}$ \\
\hline $7^{a}$ a sessão & Pais & $\begin{array}{l}\text { Significação de anseios dos pais por meio de vídeos e conceitos sobre } \\
\text { fonação e patologias vocais, e discussão sobre as mudanças percebidas } \\
\text { na dinâmica vocal das crianças }\end{array}$ \\
\hline $8^{a}$ sessão & Crianças & $\begin{array}{l}\text { Retomada da discussão sobre a fisiologia da respiração, introduzindo por } \\
\text { meio de uma dramatização a técnica de limpeza nasal e a importância da } \\
\text { respiração nasal*^ }\end{array}$ \\
\hline 9a sessão & Crianças & $\begin{array}{l}\text { Apresentação de vídeos de personagens de desenhos, discussão sobre } \\
\text { cada uma das vozes conscientização a respeito dos prejuízos do mau uso } \\
\text { e abuso vocal }{ }^{\star *}\end{array}$ \\
\hline $10^{a}$ sessão & Crianças & $\begin{array}{l}\text { Discussão sobre a musculatura laríngea e introdução de técnicas de } \\
\text { alongamento e preparação da musculatura cervical e laríngea para uma } \\
\text { fonação equilibrada** }\end{array}$ \\
\hline $11^{a}$ sessão & Crianças & $\begin{array}{l}\text { Discussão sobre mudanças voz no dia-a-dia, desenho sobre possíveis } \\
\text { mudanças na voz e encerramento com as crianças }\end{array}$ \\
\hline $12^{-\mathrm{a}} \mathrm{sec}$ & Pais & $\begin{array}{l}\text { Discussão sobre percepção de mudanças no comportamento vocal das } \\
\text { crianças e encerramento com os pais }\end{array}$ \\
\hline
\end{tabular}

Legenda: OFAs: órgãos fonoarticulatórios; CPFA: coordenação pneumofonoarticulatória; *Pais orientados a acompanhar a execução dos exercícios em casa, 3 vezes ao dia, 15 repetições cada exercício.; ${ }^{* *}$ Ao final de das sessões os exercícios eram relembrados e novamente orientados para casa durante a semana. 


\section{RESULTADOS}

O conjunto dos dados permitiu caracterizar a dinâmica vocal do grupo de crianças pré e pós-processo terapêutico grupal considerando os seguintes aspectos: frequência fundamental, intensidade vocal média, jitter, shimmer, grau geral da disfonia, rugosidade, soprosidade, tensão, pitch e loudness. As Tabelas abaixo mostram tanto a caracterização quanto a comparação a partir da aplicação de teste estatístico.

A Tabela 1 apresenta a comparação dos dados da avaliação perceptivo-auditiva, pré e pós-terapia. Foram comparadas as médias atribuídas a cada parâmetro pelo avaliador. É possível observar nos parâmetros grau geral e rugosidade houve diferença estatística entre as avaliações realizadas antes e depois do processo terapêutico grupal $(p=0,024$ e $\mathrm{p}=0,074$, respectivamente).

Tabela 1 - Comparação da análise perceptiva-auditiva da voz pré e pós terapia

\begin{tabular}{|c|c|c|c|c|c|}
\hline \multicolumn{2}{|c|}{ CAPE-V } & Média & Mediana & Desvio Padrão & p-valor \\
\hline \multirow{2}{*}{ Grau Geral } & Pré & 31,2 & 34,0 & 12,8 & \multirow{2}{*}{$0,024^{*}$} \\
\hline & Pós & 24,8 & 27,0 & 13,8 & \\
\hline \multirow{2}{*}{ Rugosidade } & Pré & 29,3 & 30,0 & 12,3 & \multirow{2}{*}{$0,074^{*}$} \\
\hline & Pós & 21,8 & 21,5 & 14,5 & \\
\hline \multirow{2}{*}{ Soprosidade } & Pré & 22,5 & 23,0 & 11,8 & \multirow{2}{*}{0,457} \\
\hline & Pós & 18,8 & 20,0 & 8,4 & \\
\hline \multirow{2}{*}{ Tensão } & Pré & 11,5 & 9,0 & 10,3 & \multirow{2}{*}{0,796} \\
\hline & Pós & 12,7 & 13,0 & 7,2 & \\
\hline \multirow{2}{*}{ Pitch } & Pré & 13,3 & 7,5 & 16,0 & \multirow{2}{*}{0,718} \\
\hline & Pós & 11,7 & 11,0 & 9,9 & \\
\hline \multirow{2}{*}{ Loudness } & Pré & 16,8 & 14,5 & 12,2 & \multirow{2}{*}{0,875} \\
\hline & Pós & 15,5 & 15,0 & 14,4 & \\
\hline
\end{tabular}

TESTE T-Student Pareado

A Tabela 2 apresenta os dados referentes à análise acústica da voz das crianças pré e pós-terapia apontando a média da frequência fundamental, o desvio padrão da frequência fundamental e a intensidade vocal média. Observa-se que, na comparação entre os dados obtidos pré e pós-terapia, não houve diferença estatística entre eles ( $p=0,288 ; p=0,254$ e $p=0,906$, respectivamente).

Tabela 2 - Comparação da análise acústica pré e pós terapia

\begin{tabular}{ccccccc}
\hline \multirow{2}{*}{ Voxmetria } & \multicolumn{2}{c}{ Média Fo } & \multicolumn{2}{c}{ Desvio Padrão F0 } & \multicolumn{2}{c}{ Intensidade Média } \\
\cline { 2 - 7 } & Pré & Pós & Pré & Pós & Pré & Pós \\
\hline Média & 229,5 & 243,6 & 12,4 & 26,4 & 61,5 & 60,9 \\
Mediana & 227,2 & 246,3 & 10,1 & 17,3 & 63,9 & 59,5 \\
Desvio Padrão & 21,6 & 23,9 & 8,5 & 24,7 & 10,8 & 6,2 \\
\hline p-valor & \multicolumn{2}{c}{0,288} & \multicolumn{2}{c}{0,254} & \multicolumn{2}{c}{0,906} \\
\hline
\end{tabular}

TESTE T-Student Pareado. Legenda: F0: frequência fundamental.

Na Tabela 3 é possível observar a complementação dos dados da análise acústica, caracterizando e comparando as atribuições dadas à jitter e shimmer, pré e pós-processo terapêutico. Em ambos os parâmetros, houve diferença estatisticamente significante entre as avaliações iniciais e finais (jitter $-p=0,079$; shimmer $-p=0,046$ ). 
Tabela 3 - Análise comparativa de jitter e shimmer pré e pós terapia

\begin{tabular}{|c|c|c|c|c|c|c|}
\hline \multirow{2}{*}{\multicolumn{2}{|c|}{ Voxmetria }} & \multicolumn{2}{|c|}{ Pré } & \multicolumn{2}{|c|}{ Pós } & \multirow{2}{*}{ p-valor } \\
\hline & & $\mathrm{N}$ & $\%$ & $\mathrm{~N}$ & $\%$ & \\
\hline \multirow{2}{*}{ Jitter } & Alterado & 5 & $83,3 \%$ & 2 & $33,3 \%$ & \multirow{2}{*}{$0,079^{*}$} \\
\hline & Normal & 1 & $16,7 \%$ & 4 & $66,7 \%$ & \\
\hline \multirow{2}{*}{ Shimmer } & Alterado & 6 & $100 \%$ & 3 & $50,0 \%$ & \multirow{2}{*}{$0,046^{*}$} \\
\hline & Normal & 0 & $0,0 \%$ & 3 & $50,0 \%$ & \\
\hline
\end{tabular}

TESTE Igualdade de Duas Proporções. Legenda: N: número de sujeitos; \%: porcentagem de sujeitos.

\section{DISCUSSÃO}

Embora ainda seja escasso o número de publicações relacionadas à atuação no grupo terapêutico fonoaudiológico com crianças disfônicas, os resultados apontam que o ganho qualitativo é central e bastante significante nesse tipo de abordagem terapêutica ${ }^{13-15}$. Autores justificam seus achados considerando que a infância é uma fase privilegiada para se trabalhar a terapêutica grupal, tanto pelo fato da criança estar em intenso processo de desenvolvimento quanto em constante aprendizado e aquisição de conceitos.

Já os dados quantitativos que evidenciam que os processos terapêuticos são eficientes com as crianças disfônicas têm se apresentado de forma restrita, no que se refere à efetividade quanto à dinâmica vocal ${ }^{16}$. Tal situação decorre de fatores históricos relacionados à atuação terapêutica nas disfonias infantis, à dúvida quanto a melhor conduta terapêutica que sempre acompanhou os profissionais que atuam nessa área ${ }^{17} \mathrm{e}$ à atitude prescritiva no sentido de "higienizar a voz" em atendimento individualizado por meio de regras que nem sempre podem ser cumpridas ${ }^{13,16}$. Devido a esses fatores, existem poucas crianças que passam por avaliação e seguem em tratamento terapêutico. Isso ocorre, dentre outro motivos, devido ao pouco conhecimento dos pais acerca dos riscos que os distúrbios da voz quando não tratados podem trazer para o desenvolvimento sócio-afetivo da criança. O conhecimento restrito dos pais gera pouca disciplina no que se refere às orientações acerca de saúde vocal ${ }^{16}$. Apesar das dificuldades do trabalho vocal na infância, o presente estudo aponta evolução em medidas quantitativas pós-processo terapêutico, buscando iniciar reflexões acerca dessa possibilidade de atuação.

A frequência fundamental e a intensidade média da voz são qualidades físicas da onda sonora ${ }^{5}$. A frequência fundamental consiste na frequência mais baixa do pulso glótico e está ligada ao número de vibrações das pregas vocais em um segundo, sendo determinada pelo tamanho, tensão e massa da cobertura das pregas vocais combinados com a pressão subglótica5,18,19. A média de frequência fundamental apresentada pelas crianças antes da terapia foi de $229,5 \mathrm{~Hz}$ e após a terapia foi de $243,6 \mathrm{~Hz}$. Alguns estudos referem que a frequência fundamental esperada na infância seria acima de $250 \mathrm{~Hz}^{5}$, e que não há diferença na frequência fundamental entre meninos e meninas. Sabe-se, ainda, que há decréscimo da $\mathrm{F}_{0}$ com o aumento da idade $^{20}$. Pensando nessa diminuição progressiva da frequência fundamental com a idade, um estudo com crianças de seis a oito anos realizado em Belo Horizonte, mostrou que a média para todas as faixas etárias foi de $249,71 \mathrm{~Hz}$, mas para crianças da faixa etária de oito anos, essa média diminuiu para $235,77 \mathrm{~Hz}^{20}$. Nossos resultados pós-terapêuticos estão mais próximos de tais valores.

A redução da frequência fundamental é comum em lesões de massa ou alterações na mobilização da mucosa das pregas vocais 5 . O discreto aumento nos valores referentes a ela no momento pós-terapia pode ter relação com a redução da rugosidade e com o desenvolvimento de uma melhor mobilização de mucosa das PPVV.

Em relação à avaliação perceptivo-auditiva, observa-se que no momento pré-terapia, a rugosidade era o parâmetro mais alterado. No entanto, as medianas referentes ao grau global da disfonia e à rugosidade obtidas pelas crianças na CAPE- $V$ antes da terapia, 34 e $30 \mathrm{~mm}$, respectivamente, apontam que, em geral, as crianças iniciaram processo terapêutico apresentando disfonia de grau discreto. Estudo utilizando a escala CAPE-V mostrou que até $35,5 \mathrm{~mm}$ é possível que se esteja diante da variabilidade normal da voz, ou seja, a pequenos desvios que não são considerados como distúrbios vocais ${ }^{21}$. No momento pós-terapia, os valores referentes às mesmas medianas, 27 e $21 \mathrm{~mm}$, respectivamente, indicam redução do grau global da disfonia e da rugosidade, reforçando a efetividade do processo terapêutico.

Não foram observadas alterações significantes de pitch, loudness e tensão em nenhum dos 
momentos de avaliação. Tais achados podem ter relação com o grau global da disfonia e os graus de rugosidade e soprosidade das crianças no início da terapia. Como, em geral, as crianças apresentavam alterações de qualidade vocal de grau discreto, os demais parâmetros não sofreram grandes interferências.

As crianças muitas vezes convivem com modelos vocais inadequados, tendendo a imitá-los, e fixar esses padrões errôneos ${ }^{15}$, o que pode ter contribuído para o desenvolvimento da disfonia. Após a terapia, a conscientização das crianças em relação aos hábitos vocais inadequados, aos padrões vocais adequados, a interferência dos fatores biopsicofísicos e aos prejuízos gerados pela imitação vocal ${ }^{21}$, pode ter facilitado, em geral, a modificação dos parâmetros vocais.

A intensidade vocal média apresentada pelas crianças antes da terapia foi de $61,5 \mathrm{~dB}$, e após a intervenção foi de $60,9 \mathrm{~dB}$, não havendo diferença estatisticamente significante entre elas. A intensidade da fala de um indivíduo é um parâmetro vocal definido e fixado durante a primeira infância e por isso dificilmente pode ser modificado, mesmo terapeuticamente. Seu controle está relacionado à consciência da dimensão exata do outro e ao refinado domínio de projeção no espaço ${ }^{22}$. Nossos resultados corroboram com os achados de um estudo que obteve intensidade vocal em torno de $64 \mathrm{~dB}$ para crianças, durante conversação habitual ${ }^{23}$. Assim, em ambos os momentos de avaliação (pré e pós-terapia), o parâmetro intensidade vocal encontrava-se dentro dos padrões de normalidade no grupo estudado.

Conforme mencionado, as medidas de ruído avaliadas e consideradas neste estudo foram jittere shimmer, que são parâmetros acústicos de perturbação do sinal vocal, bastante investigados em crianças ${ }^{5,18,24}$. O jitter é definido como uma variável de curto tempo (ciclo-a-ciclo) na frequência fundamental dentro da amostra de voz analisada, medida entre ciclos glóticos vizinhos e expressa a diferença quantitativa entre um período e seu antecessor ou sucessor imediato ${ }^{24}$. Já o shimmer é a variável de curto-tempo na amplitude da amostra de voz analisada, e representa as alterações irregulares na amplitude dos ciclos glóticos, de um ciclo a outro ${ }^{24}$. Há concordância na literatura que a avaliação de tais aspectos é importante durante o processo de análise vocal ${ }^{5,24}$. Os padrões de normalidade dessas medidas variam de acordo com o software utilizado. Observa-se que, entre os dados coletados em pré e pós-processo terapêutico, houve diferença estatística para jitter e shimmer, evidenciando a melhora de tais aspectos. Em indivíduos com vozes adaptadas, os valores do jitter e shimmer são baixos, contudo, na presença de lesões de pregas vocais, há uma maior aperiodicidade de vibração e de amplitude das ondas, que se expressa em valores mais elevados de tais medidas ${ }^{18}$. Portanto, atribuí-se a melhora desses parâmetros a uma provável maior periodicidade de vibração das pregas vocais, devido a um maior equilíbrio entre as forças mioelásticas da laringe e as forças aerodinâmicas pulmonares.

Estudo que avaliou, tratou e reavaliou, crianças disfônicas, em grupo terapêutico fonoaudiológico não privilegiou os aspectos quantitativos de evolução ${ }^{13}$ Os autores referem que houve evoluções qualitativas, proporcionadas pelo espaço gerado no grupo para o processo de negociação de significados. Tais negociações promoveram modificações quanto à percepção sobre saúde vocal e o conhecimento sobre os cuidados para manter os padrões vocais normais ${ }^{14}$. No presente estudo, avança-se tal reflexão, observando que houve modificações reais das características vocais. Tais mudanças puderam ser percebidas por meio de análise perceptivo-auditiva e acústica.

Crianças disfônicas, em geral, apresentam vozes com qualidade rugosa-soprosa, tempos máximos fonatórios reduzidos, pitch e loudness inadequados e incoordenação penumofonoarticulatória ${ }^{16}$. A literatura refere que a rouquidão pode estar associada com o uso intenso e inadequado da voz, sendo também, o sintoma mais comum nos quadros de disfonia decorrente de abuso vocal, uso inadequado da voz ou associado às alterações de vias aéreas superiores ${ }^{5}$. Uma das mais importantes causas de alterações vocais na infância é o abuso vocal, que pode ser modificado com o conhecimento sobre higiene vocal ${ }^{25}$. No grupo terapêutico fonoaudiológico tal abordagem ocorreu a partir de troca de experiências entre as crianças. Não houve foco exclusivo nas repetições de sequencias de exercícios, porém, deixa-se claro que as técnicas certamente foram importantes para a modificação dos parâmetros vocais das crianças. A literatura é muito restrita quanto a estudos que mostram a evolução dos parâmetros vocais após fonoterapia em grupo.

Em relação ao público jovem e adulto, um estudo de terapia fonoaudiológica grupal em estudantes de pedagogia que apresentavam disfonia discreta, indicou que o trabalho grupal fonoaudiológico com inclusão de exercícios possibilitou aos participantes melhora da qualidade vocal, dos aspectos laríngeos e também diminuição no numero de sintomas após a intervenção ${ }^{26}$. Observa-se, portanto, que o treinamento vocal tem se mostrado efetivo com o público jovem e adulto. Nesse sentido, novos estudos acerca do processo terapêutico grupal junto a 
crianças disfônicas devem ser desenvolvidos, a fim que se amplie as discussões referentes ao tema.

O trabalho com o grupo terapêutico se mostrou uma importante forma de trabalho fonoaudiológico já que atua nos aspectos da promoção da saúde construindo com os sujeitos conceitos e noções de autocuidado e educação para saúde. Além disso, oferece possibilidades de trabalho clínico-terapêutico por meio da prática de exercícios vocais, que nesse estudo mostraram-se eficazes pós-terapia.

Além disso, o trabalho foi realizado de forma lúdica, fazendo com que as crianças envoltas em um contexto de brincadeira se desprendessem da terapia puramente motora, com exercícios que acabam se tornando pouco atraentes para as crianças. Essa terapia repetitiva faz com que as crianças, muitas vezes não persistam na realização do procedimento, e devido a pouca consciência sobre as vantagens dos procedimentos, não vejam vantagens reais na terapia, pois muitas vezes deixa-se de lado o trabalho com a conscientização de suas vantagens e da implicação na sua interação com os demais, bem como em outros aspectos biopsicossociais ${ }^{2,16}$.

A falta de preocupação dos pais e professores com a voz da criança, vendo as alterações como um processo normal do desenvolvimento infantil é uma grande barreira para o atendimento às crianças disfônicas ${ }^{27}$. Mesmo após a conclusão diagnóstica de disfonia, é possível observar pouco interesse dos pais e de algumas crianças pelo processo de reabilitação vocal, já que nem sempre os impactos da disfonia são percebidos na vida diária ${ }^{17,27}$. Neste trabalho, foi possível observar a importância da demanda dos pais, incentivando seus filhos a participarem, e a mudarem seus comportamentos para adequarem os padrões vocais. Os encontros com os pais esclareceram muitas dúvidas e serviram como um espaço de troca de informações, possibilitando a melhor percepção dos pais com relação aos danos que os problemas vocais podem trazer para o desenvolvimento da capacidade de comunicação adequada ${ }^{17,27}$.

Diversos estudos realizados com grupos terapêuticos fonoaudiológicos mostram o quanto estes podem favorecer transformações nos indivíduos, permitindo aos seus integrantes a partilha do processo vocal de si e dos outros, propiciando um espaço de negociação e emergência de condições de exploração e vivência da voz de cada um ${ }^{2,13-16}$. A atuação fonoaudiológica dentro da perspectiva social da voz é eficaz, pois o grupo permite ao paciente perceber sua voz na interação com as pessoas $^{28}$. Nesse contexto, atenta-se para o fato de que o grupo terapêutico pode ser utilizado como uma estratégia de saúde coletiva, não apenas como procedimento clínico, pois permite resultados efetivos na saúde vocal de um grupo de crianças, considerando o funcionamento grupal e individual simultaneamente.

Sugere-se a realização de novos estudos, envolvendo processos terapêuticos em grupo que incorporem em sua metodologia de trabalho estratégias facilitadoras referentes aos diferentes parâmetros vocais (qualidade vocal, pitch, loudness, etc.). Isso contribuirá para o delineamento das ações fonoaudiológicas junto a crianças disfônicas e para a efetividade de nossa atuação junto a essa população.

\section{CONCLUSÃO}

A terapia fonoaudiológica grupal promove modificações na dinâmica vocal de crianças disfônicas, no que se refere aos parâmetros perceptivo-auditivos e acústicos. 


\begin{abstract}
This study aims at featuring the dynamic vocals of dysphonic children before and after speech therapy group by assessing perceptual and acoustic voice. Participants were six children, two boys and four girls, aged between seven and ten year old with a diagnosis of functional dysphonia or functional-organo one. Children were submitted to an interview, perceptual analysis and acoustic voice before and after weekly group therapy process, which amounted to twelve sessions of forty minutes each. As therapeutic strategies we have been proposed drama activities, drawings, games, preparation of panels and vocal exercises conducted (collectively and playful). We tried to exchange experiences among group members, the joint construction of knowledge about voice production and vocal health, and through the direct performance of techniques and exercises. Data were analyzed using a significance level of 0.10 . As for the roughness parameters and the overall level of dysphonia perceptual assessment, there were differences between the assessments before and after the therapy process group ( $p=0.024$ and $p=0.074$, respectively). Regarding the acoustic voice analysis before and after therapy, there was no difference in fundamental frequency and vocal intensity mean $(p=0.288$ and $p=0.906$, respectively). As for the measures of noise, jitter and shimmer, there was no difference among the initial and final evaluations ( $p=0.079$ and $p=0.046$, respectively). The speech therapy group promotes changes in the dynamic vocals of dysphonic children, in relation to the perceptual and acoustic parameters.
\end{abstract}

KEYWORDS: Dysphonia; Voice Disorders; Speech Therapy; Group Practice; Child

\section{REFERÊNCIAS}

1. Chun RYM, Servilha EMA, Santos LMA, Sanches $\mathrm{MH}$. Promoção de Saúde: o conhecimento do aluno de jornalismo sobre sua voz. Distúrb Comun. 2007; 19(1):73-80.

2. Leite APD, Panhoca I, Zanolli ML. Distúrbios de voz em crianças: o grupo como possibilidade de intervenção. Rev Dist Comun. 2008 Dez; 20(3):339-47.

3. Souza APR, Crestani AH, Vieira CR, Machado FCM, Pereira LL. Speech and language therapy and the group: clinical and coletive health sources. Rev. CEFAC. 2011; 13(1):140-51.

4. Gasparini G, Azevedo R, Behlau M. Experiência na elaboração de estórias com abordagem cognitiva para tratamento de disfonia infantil. R. Ci. méd. biol. 2004; 3(1): 82-8.

5. Behlau M. Voz: o livro do especialista II. Rio de Janeiro: Revinter, (2) 2005.

6. Takeshita TK, Aguiar-Ricz L, Isaac ML, Ricz $\mathrm{H}$, Anselmo-Lema W. Comportamento Vocal de Crianças em Idade Pré-escolar. Arq. Int. Otorrinolaringol. 2009; 13(3):252-8.

7. Cielo CA, Cappellari VM. Tempo Máximo de Fonação de Crianças Pré-escolares. Rev. Bras. Otorrinolaringol. 2008; 74(4):552-60.

8. Jotz GP, Cervantes O, Sattani FAP, Angelis EC. Acoustic measures for the detection of hoarseness in children. Arq Int Otorrinolaringol. 2006; 10(1):14-20.
9. Santos MAO, Moura JMP, Duprat AC, Costa HO, Azevedo BB. A Interferencia da Muda Vocal nas Lesões Estruturais das Pregas Vocais. Rev Bras Otorrinolaringol. 2007; 73(2):226-30.

10. Speyer R. Effects of voice therapy: a sistematic review. J Voice. 2008; 22(5):565-80.

11. Dornelles S, Jotz GP, Guilherme A. Correlação entre avaliação perceptiva auditiva e nasofibroscopia em crianças sem queixa vocal. Rev da AMRIGS. 2007; 51(2):121-7.

12. American Speech-Language-Hearing Association. Consensus auditory-perceptual evaluation of voice CAPE-V [text on the Internet]. Disponível em: http:// www. asha.org/NR/rdonlyres/79EE699E-DAEE4E2C-A69E-C11BDE6B1D67/0/22560_1.pdf. Acesso em: 12/10/2010.

13. Leite APD, Panhoca I, Zanolli ML. Distúrbios de voz em crianças: o grupo como possibilidade de intervenção. Rev Dist Comun. 2008; 20(3):339-47.

14. Vilela FCA, Ferreira LP. Voz na clínica fonoaudiológica: grupo terapêutico como possibilidade. Rev Dist Comun. 2006; 18(2):235-43. 15. Penteado RZ, Camargo AMD, Rodrigues CF, Silva CR, Rossi D, Silva JTC, et al. Vivência de voz com crianças: análise do processo educativo em saúde vocal. Rev Dist Comun. 2007; 19(2):237-46.

16. Leite APD, Panhoca I. Avaliação Vocal em Crianças Disfônicas antes e após Intervenção Fonoaudiológica em Grupo. Rev Salus. 2008; 2(1): 69-74. 
17. Martins RHG, Trindade SHK. A criança disfônica: diagnóstico, tratamento e evolução clínica. Rev Bras Otorrinolaringol. 2003; 69(6): 801-6.

18. Greene MCL, Mathieson L. The voice and its disorders. San Diego: Singular Publishing. 1989; 5. 19. Zhang K, Siegmund T, Chan RW, Fu M. Predictions of fundamental frequency changes during phonation based on a biomechanical model od the vocal fold lamina propria. J Voice. 2009; 23(3):277-82.

20. Braga JÁ, Oliveira DSF, Sampaio TMM. Frequência fundamental da voz de crianças. Rev CEFAC. 2009; 11(1):119-26.

21. Yamasaki RK, Leão SHS, Madazio G, Padovani $M$, Azevedo RR, Behlau, MS. Correpondência entre escala analógico-visual e escala numérica na avaliação perceptivo-auditiva de vozes. In: Anais do $16^{\circ}$ Congresso Brasileiro de Fonoaudiologia. Campos do Jordão; 2008.

22. Zaffari R, Feijó A, Scalco M. Estudo da intensidade vocal habitual em uma amostra de crianças de 4 a 6 anos. Pró-fono. 1999; 11(1):94-9. 23. Vasconcelos LR. Análise da intensidade no campo dinâmico vocal de acordo com o sexo e a idade [monografia]. São Paulo (SP): CEV - Centro de Estudos da Voz; 1994.

24. Smits I, Ceuppens P, DeBodt MS. A Comparative Study od Acoustic Voice Measurements by Means of Dr. Speech and Computerized Speech Lab. J Voice. 2006; 19 (2): 187-93.

25. Pereira JG, Cervantes $O$, Abrahão $M$, Parente SFA, Carrara-de-Andelis E. Noise-to-harmonics ratio as an acoustic measure of voice disorders in boys. J. Voice. 2002; 16(1):28-31.

26. Simberg S, Sala E, Tuomainem J, Sellman J, Rönnema AM. The effectiveness of group therapy for students with mild voice disorders: a controlled clinical trial. J Voice. 2006; 20(1):97-109.

27. Teixeira MZM, Trezza EMC, Behlau M. Opinião dos pais sobre a voz de seus filhos de 5 a 12 anos. Rev. Paul. Pediatria. 2003; 21(2): 68-75.

28. Chun RYS. Voz profissional: repensando conceitos de prática na promoção da saúde vocal. In: Ferreira LP, Andrada e Silva MA, organizadoras. Saúde vocal: práticas fonoaudiológicas. São Paulo: Roca; 2002; 1:19-31.
http://dx.doi.org/10.1590/S1516-18462012005000056

RECEBIDO EM: 02/08/2011

ACEITO EM: 04/12/2011

Endereço para correspondência:

Vanessa Veis Ribeiro

Rua Souza Naves, 227, Jardim Califórnia

Irati - PR

CEP: 84500-000

E-mail: vanessaribeirooo@ hotmail.com 